\title{
Lane-Change Model and Tracking Control for Autonomous Vehicles on Curved Highway Sections in Rainy Weather
}

\author{
Tao Peng, ${ }^{1,2}$ Li-li Su, ${ }^{3}$ Zhi-wei Guan $\mathbb{D}^{4}{ }^{4}$ Hai-jing Hou, ${ }^{1}$ Jun-kai Li, ${ }^{5}$ Xing-liang Liu, ${ }^{3}$ \\ and Yi-ke Tong ${ }^{6}$ \\ ${ }^{1}$ College of Automobile and Transportation, Tianjin University of Technology and Education, Tianjin 300222, China \\ ${ }^{2}$ The State Key Laboratory of Automotive Safety and Energy, Beijing 100083, China \\ ${ }^{3}$ China Automotive Technology and Research Center Co., Ltd., Tianjin 300300, China \\ ${ }^{4}$ Tianjin Sino-German University of Applied Sciences, Tianjin 300350, China \\ ${ }^{5}$ Zhongshan Technician College, Zhongshan 528400, China \\ ${ }^{6}$ College of Mechanical Engineering, Zhejiang University of Technology, Hangzhou 310014, China
}

Correspondence should be addressed to Zhi-wei Guan; zhiwguan@163.com

Received 2 July 2020; Revised 21 September 2020; Accepted 10 October 2020; Published 25 November 2020

Academic Editor: Bin Yu

Copyright (c) 2020 Tao Peng et al. This is an open access article distributed under the Creative Commons Attribution License, which permits unrestricted use, distribution, and reproduction in any medium, provided the original work is properly cited.

In this study, we propose an adaptive path planning model and tracking control method for collision avoidance and lane-changing manoeuvres on highways in rainy weather. Considering the human-vehicle-road interaction, we developed an adaptive lane change system that consists of an intelligent trajectory planning and tracking controller. Gaussian distribution was introduced to evaluate the impact of rain on the pavement characteristics and deduce adaptive lane-change trajectories. Subsequently, a scorebased decision mechanism and multilevel autonomous driving mode that considers safety, comfort, and efficiency were proposed. A tracking controller was designed using a linearised model predictive control method. Finally, using simulated scenarios, the feasibility and effectiveness of the proposed method were demonstrated. The results obtained herein are a valuable resource that can be used to develop an intelligent lane change system for autonomous vehicles and can help improve highway traffic safety and efficiency in adverse weather conditions.

\section{Introduction}

Weather is an important factor that affects traffic and road safety. Nearly, $75 \%$ of all traffic accidents are caused due to slippery pavement and around $47 \%$ of traffic accidents occur on the rainy days of the year [1]. Rainfall has a significant impact on vehicle manoeuvrability and is of particular importance in path planning and control mechanisms in autonomous vehicles, on account of the complex environment perception and manipulation coupling, along with lower visibility and pavement adhesion. Therefore, an adaptive lane change system that can achieve safe lane changes in rainy weather is an important development goal.

An intelligent lane change system for autonomous vehicles must address two critical issues-path planning and tracking control. Path planning for lane changing is a localised motion that occurs in real-time and is closely related to the vehicle dynamics and parameters relating to the external environment, such as the decision time and adhesion coefficient of pavement. Tracking control is based on the goal defined by path planning and must provide tracking accuracy and robust stability. Over the past few years, a significant amount of progress has been achieved in this field [2-4].

Classical path planning strategies using search-based and random sampling methods, such as $\mathrm{A} *$ [5], $\mathrm{D} *$ [6], and rapidly-exploring random tree (RRT) $[7,8]$, have been widely used in the field of robotics. Stentz demonstrated an extension to the $\mathrm{D} *$ algorithm that can significantly reduce the total time required for path planning. Search-based methods achieve path planning using the target-oriented heuristic method, and the $\mathrm{D} *$ algorithm is better suited to 
dynamic environments [9]. However, it is more suited to single and discrete node states than to continuous vehicle motion. Consequently, Frazzoli et al. proposed a randomised motion planning architecture for dynamic systems with fixed and moving obstacles, which describes a real-time motion-planning algorithm by search tree. Nevertheless, large oscillations and uncertain convergence rates exist in the planning path, and the optimality cannot be guaranteed [10]. In recent years, with the development of artificial intelligence (AI) technology, numerous advanced swarm intelligence methods have been applied to path planning in autonomous vehicles, including ant colony, genetic, and artificial neural network algorithms. Liu proposed an improved ant colony algorithm to optimise path planning [11]. Engedy et al. verified that an artificial neural network algorithm can be used to control an autonomous vehicle $[12,13]$. However, AI algorithms are complex, and the problem of slow convergence is extremely prominent. Moreover, if the dataset is insufficient, the neural networks may cause deviations. Therefore, autonomous vehicles tend to employ more practical path-planning methods, such as the potential field and optimisation curves [14]. The basic idea behind the potential field is to abstract the driving environment of a host vehicle into a virtual potential field by defining a potential energy function to ensure collision avoidance and path optimisation [15, 16]. Although the algorithm can clearly describe progressive states between related vehicles, it is complex to compute. Consequently, the real-time rendering and control of potential energy functions cannot be achieved easily. In comparison, optimisation curves are more attractive on account of their real-time applicability and simplified vehicle dynamics, with typical methods, including splines, Bézier curves, and geometric functions. Gomez et al. generated an effective collision-free path based on $\beta$-spline curves [17]. You et al. introduced tracking-planning methods using polynomials for lanechange manoeuvres $[18,19]$. Kuniaki et al. presented an optimised path generation method based on Bézier curves for anticollision behaviour [20]. Geometric methods have attracted significant attention as they provide real-time computation, and the constant offsets are a good solution to the problem of occupant comfort during vehicle lane changes. Wang et al. proposed trapezoidal and sine functions to describe lane-change trajectories [21]. A path-based sine function is easy to calculate and has continuous curvature, but the maximum values of the lateral acceleration of the vehicle exist at the beginning and end of the path, which is inconsistent with reality. Polynomial functions are idealised, and yaw motion is neglected. Therefore, although geometric curves can be parameterised easily, it is difficult to describe a clear link between actual driving and vehicle characteristics.

Based on the lane-change path planning method, the decision system determines a reasonable path, and a control module executes the selected lane-change trajectory. A significant amount of research has been conducted on control methods [22, 23]. Classical control methods are based on the steering mechanism. MacAdam proposed an optimal single-point preview steering controller to ensure lateral trajectory tracking, and Sharp et al. designed a multipoint preview steering controller using a linear quadratic regulator (LQR) to improve the control effect [24-26]. Meanwhile, PID control is one of the most widely used algorithms. Akash et al. designed a nested controller for autonomous vehicles using double-integral action to minimise trajectory tracking errors [27, 28]. Although these controllers employ simple and easy to implement structures, they cannot be used in applications that require high tracking accuracy. Sangeetha et al. developed an adaptive integral backstepping control approach for unmanned aerial vehicles to optimise lateral tracking, based on the Lyapunov theorem [29]. Wang et al. presented a sliding mode variable structure controller using an automatic steering control algorithm [30]. These classical control methods are widely used in autonomous vehicles on account of their high theoretical maturity. However, owing to the high accuracy requirements of the model, the control effect under complex nonlinear constraints cannot be guaranteed. To adapt to vehicle state time variants and multiconstraint sets, such as actuator saturation of the steering system or roads with unknown curvature and uncertain lateral wind forces, fuzzy and model predictive control (MPC) have been applied to improve the stability and robustness. Nguyen et al. proposed a new constrained Takagi-Sugeno fuzzy model-based control method using a fuzzy Lyapunov control framework to perform automatic lane-keeping under multiple system constraints [31]. However, fuzzy rules rely on experience, and the relevant theories must be further improved. In contrast, an MPC controller can deal with uncertainties in its prediction and optimisation ability. Che et al. presented a robust MPC approach to control front steering [32]. Bascetta et al. developed a novel methodology based on a linear fractional transform (LFT) formulation of the system dynamics [33]. Still, linear control algorithms are inadequate for actual vehicles, especially for those working in highly nonlinear regions. Therefore, considering the complexity of vehicle-driving scenarios and states, Mario et al. presented a nonlinear MPC and moving horizon estimation for highly nonlinear, constrained, unstable, and fast dynamic systems. The performance of the proposed controller offered better state prediction and tracking on a low-friction icy road [34].

Traditional methods of path planning and control have played a positive role in the study of autonomous vehicles. However, most existing models only consider vehicle dynamics and seldom consider the impact of the characteristics of an intelligent driver and the road on driving safety in rainy weather. Furthermore, previous studies have focussed primarily on straight roads, without paying much attention to curved sections. Vehicle lane changing on a curved highway to avoid a collision is a relatively complex working condition that synchronously involves both steady-state and transient steering, coupled with a wide range of real-time state changes. Therefore, it is prone to instability and can cause chain rear-end collisions, especially in rainy weather.

There are three main considerations in this scenario: the first is to determine the safe distance for lane changes on a typical dangerous curved and slippery road section, which clarifies the influence of the road environment on active 
obstacle avoidance and path planning; the second involves a method to generate an intelligent decision, called hierarchical scoring, through which the system can make an optimal driving decision, considering multiple and conflicting objectives; the third is a real-time trajectory planning and following architecture that is designed based on different lane-change scenarios. Overall, we present a systematic approach from behavioural decision making and path planning to control execution for autonomous vehicles, based on Gaussian distribution, scoring, and convex optimisation, such that optimal paths and velocity profiles are generated to safely execute vehicle movement. This study can serve as a reference to develop the environmental suitability of an autonomous vehicle control system and improve the safety and efficiency of the advanced transportation system.

This study considers lane changing in an autonomous vehicle on a curved highway in rainy weather, which is a relevant real life situation. The rest of this paper is laid out as follows: Section 2 provides a brief description of the influence of rainfall on lane change behaviour; the lane change model is presented and validated in Section 3; Section 4 describes the design of a decision mechanism for collision avoidance and a controller using model predictive control (MPC); Section 5 lists the major conclusions; the conflicts of interest and acknowledgements are listed in Section 6 and Section 7 , respectively.

\section{Influence Analysis of Rainfall on Lane- Change Behaviour}

2.1. Driving Behaviour. tRainfall, especially moderate to heavy rainfall, reduces visibility, blurring the sight of the driver and increasing response time. According to [35], there is a clear decrease in the frequency of speeds $>110 \mathrm{~km} / \mathrm{h}$ in the slow lane in rainy conditions, and the median speed in the fast lane decreases by about $20 \%$ with moderate rainfall. From a microscopic standpoint, this indicates that drivers reduce their speeds in adverse weather conditions and increase their headway and spacing, as shown in Figure 1. Considering the environmental adaptability of drivers and passengers, autonomous vehicles must be able to adopt similar driving modes to humans on rainy days.

2.2. Road Adhesion Characteristics. In rainy weather, a water film layer exists between the tyre and the pavement, decreasing the road adhesion coefficient, maximum lateral force, and brake force. The thickness of the water film is mainly affected by the rainfall intensity, slope length, slope angle, and roughness of the road surface [36]:

$$
h=0.1258 \times l^{0.6715} \times i^{-0.3147} \times q^{0.7786} \times T D^{0.7261},
$$

where $h$ is the thickness of the water film ( $\mathrm{mm}), l$ is the slope length (m), $i$ is the slope angle (\%), $q$ is the rainfall intensity $(\mathrm{mm} / \mathrm{min})$, and TD is the roughness of the road surface $(\mathrm{mm})$. The road adhesion coefficient is closely related to vehicle speed and is calculated as

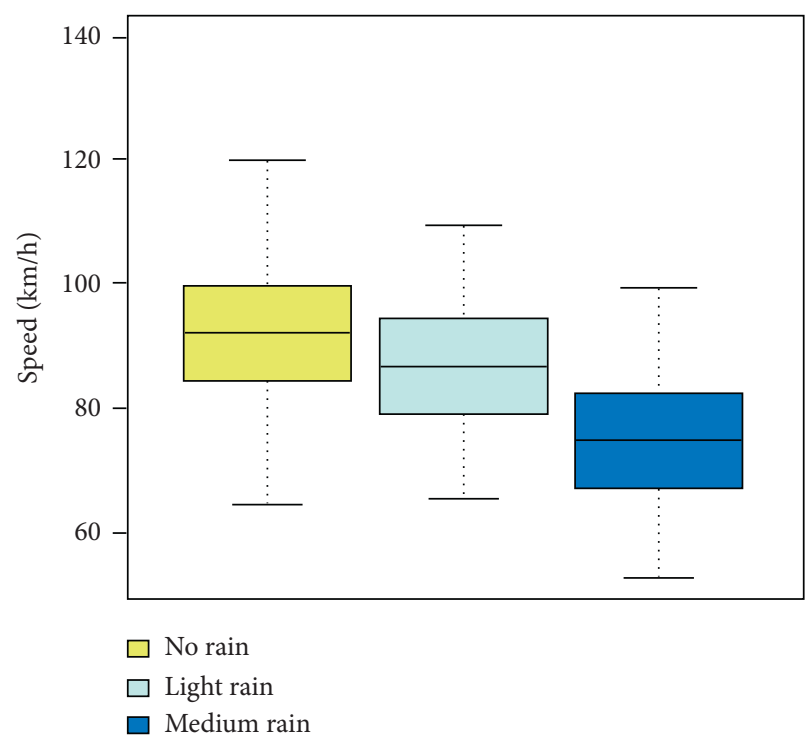

FIgURE 1: Distribution box diagram of vehicle speed in various rainfall conditions.

$$
\mu=0.9458-0.0057 \mathrm{~V}-0.0108 h,
$$

where $\mu$ is the adhesion coefficient of the road, $V$ is the vehicle speed $(\mathrm{km} / \mathrm{h})$, and $h$ is the thickness of the water film (mm).

Setting $V=108 \mathrm{~km} / \mathrm{h}, l=50 \mathrm{~m}, i=3 \%, T D=0.55 \mathrm{~mm}$, and $q=1.0 \mathrm{~mm} / \mathrm{min}$, the road adhesion coefficient is calculated to be around 0.53 , which is used as a reference herein.

2.3. Vehicle Dynamics. Rain has a significant influence on the adhesion coefficient of the road and the vehicle tyres, which can decrease by about $60 \%$. As a result, the tyre saturated lateral force cannot supply the vehicle steering centrifugal force. Consequently, sideslip is more to likely to occur while steering on a highway. In general, the vehicle acceleration threshold in rainy conditions is lower and can be calculated as

$$
a_{\max }=\mu g,
$$

where $a_{\max }$ is the maximum vehicle acceleration.

The typical performance when vehicle sideslip occurs is a sharp turn, as shown in Figure 2.

\section{Safe Lane-Change Model for Collision Avoidance}

3.1. Vehicle Lateral Dynamics. The plane movement of a vehicle lane-change manoeuvre on a curved road is shown in Figure 3. Unlike straight driving, there are initial and final turns at the beginning and end of the curve, respectively. The entire driving process can be divided into three steps, namely, initial fixed radius steering, sinusoidal steering, and final fixed radius steering.

As shown in Figure 3, the vehicle dynamics can be defined by both a ground coordinate system $X O Y$ as well as a 


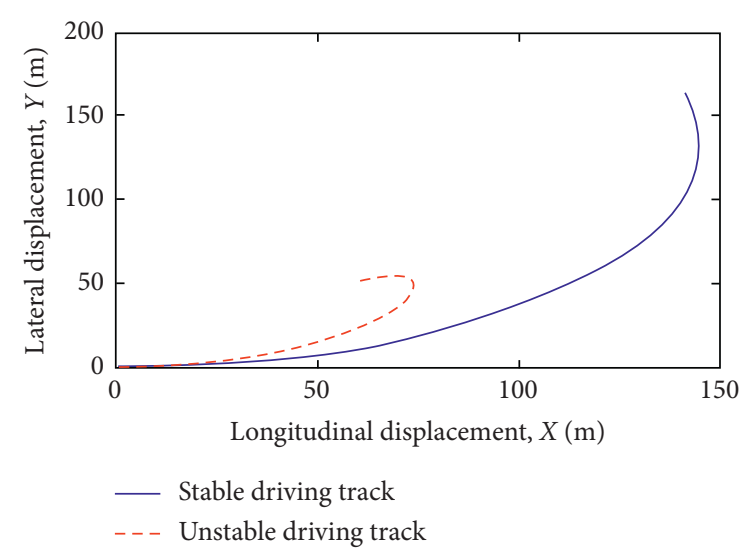

Figure 2: Comparison of vehicle driving tracks on normal and slippery roads.

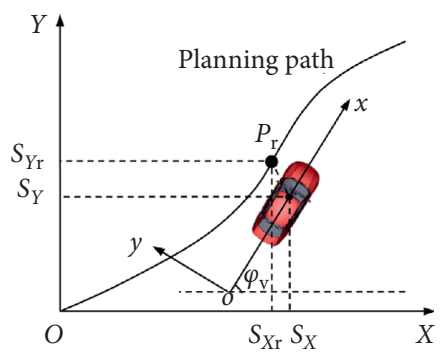

$X O Y$ - ground coordinate system xoy- vehicle coordinate system

$S_{X}$ - longitudinal ordinate of vehicle in $X O Y$

$S_{Y}$ - lateral ordinate of vehicle in $X O Y$

$\varphi_{v}$ - vehicle yaw angle

$P_{\mathrm{r}}$ - reference point of the planning path

$S_{X r}$ - longitudinal ordinate of reference point in XOY

$S_{Y \mathrm{r}}$ - lateral ordinate of reference point in $X O Y$

Figure 3: Plane movement of vehicle lane-change on curved road.

vehicle coordinate system xoy. The vehicle moves in a variable curvature motion while turning and changing lanes. Reference [37] has shown that a sine wave-shaped steeringwheel movement can describe a lane change manoeuvre. Moreover, the steering angle when driving on a curved road with constant curvature is fixed. Therefore, for a lanechanging manoeuvre on a curved road, the front wheel steering angle can be approximated as

$$
\delta_{\mathrm{f}}(t)=\delta_{\mathrm{f} 0}(t)+\delta_{\mathrm{fc}}(t)=C+A \sin (2 \pi f t),
$$

where $\delta_{\mathrm{f}}$ is the front wheel angle; $\delta_{\mathrm{fo}}$ is the turning angle, which can be considered to be a fixed value with invariant curvature; $\delta_{\mathrm{fc}}$ is the steering angle for a simple lane-change; $A$ and $f$ are the amplitude and frequency of $\delta_{\mathrm{fc}}$, respectively; $t$ is the driving time; and $C$ is a constant value adapted to the road curvature.

The steering inputs can be divided into two separate inputs: steady state cornering with fixed radius and transient steering with variable radii. Based on this idea, the vehicle lateral dynamics can be integrated by the turning motion and the lane changing motion, and the vehicle lateral velocities in $X O Y$ are

$$
V_{Y}(t)=V_{Y 0}(t)+V_{Y c}(t),
$$

where $V_{Y}$ is the synthetic vehicle lateral velocity; $V_{Y 0}$ and $V_{Y c}$ are the lateral velocities of turning and lane changing, respectively; and $t$ is the vehicle driving time.

In general, the minimum radius of a curve on highways in plains and hilly areas is more than $600 \mathrm{~m}$. Thus, the road curvature and vehicle yaw angle while changing lanes at high speed on a curved highway section are relatively small. Introducing the improved Gaussian distribution from [21], the vehicle lateral velocities can be calculated as

$$
\left\{\begin{array}{l}
V_{Y}(t)=V_{Y 0}(t)+V_{Y c}(t), \\
V_{Y 0}(t)=\frac{\left(a_{x}^{2} t^{3}+2 a_{x} v_{x 0} t^{2}+v_{x 0}^{2} t\right)}{R_{0}}, \\
V_{Y c}(t)=\frac{d}{\sqrt{2 \pi} \sigma_{y}} \exp \left(\frac{-\left(t-\mu_{y}\right)^{2}}{2 \sigma_{y}^{2}}\right),
\end{array}\right.
$$

where $R_{0}$ is the curve radius, defined as a constant value on a regular curve; $v_{x 0}$ is the vehicle longitudinal initial velocity in xoy; $a_{x}$ is the longitudinal acceleration; and $d, \mu_{y}$, and $\sigma_{y}$ are fitting coefficients, which are defined as

$$
\left\{\begin{array}{l}
d=B, \\
\mu_{y}=t_{0}+\frac{1}{(2 f)}+t_{\mathrm{d}}, \\
P=P\left\{\mu_{y}-\lambda \sigma_{y}<t<\mu_{y}+\lambda \sigma_{y}\right\}=\Phi(\lambda), \\
\sigma_{y}=\frac{\left((1 / f)+2 t_{\mathrm{d}}\right)}{(2 \lambda)},
\end{array}\right.
$$

where $B$ is the width of each lane, $f$ is the steering frequency, $t_{0}$ is the decision time of the intelligent system, $t_{\mathrm{d}}$ is the response delay time of the lateral velocity determined by the vehicle dynamics, and $P$ and $\lambda$ are the probability coefficients. $\lambda$ is generally set to $4 \sim 6$ to ensure a high probability of lane change completion in a steering cycle. In real-world scenarios, it can be obtained by accurately fitting the lateral velocity to vehicle dynamics and scenarios.

3.2. Vehicle Longitudinal Dynamics. Generally, a vehicle brakes or changes lanes to avoid a colliding with an obstacle or a slower vehicle. Changing lanes is better than braking as it is more efficient. In addition, owing to the high speeds and lower adhesion coefficient on highways during rainy weather, a longer safe distance should be taken to avoid collisions. Therefore, a combination of lane changing and longitudinal speed control is a safer and more efficient alternative. Two lane changing modes with constant speed and deceleration are discussed herein. 
On account of the small yaw angle of the vehicle (generally, $<10^{\circ}$ ), the vehicle longitudinal velocity in the ground coordinate system $\mathrm{XOY}$ is regarded as the same as that in the vehicle coordinate system xoy. The vehicle longitudinal velocity and displacement in the ground coordinate system $X O Y$ at constant speed and deceleration are

$$
\begin{aligned}
& \begin{cases}V_{X 1}(t)=v_{x 0}, & a_{x}=0, \\
S_{X 1}(t)=v_{x 0} t, & \end{cases} \\
& \begin{cases}V_{X 2}=v_{x 0}+a_{x}\left(t-t_{0}-t_{\mathrm{b}}\right), & a_{x}<0, \\
S_{X 2}=v_{x 0}\left(t_{0}+t_{\mathrm{b}}\right)+\frac{1}{2} a_{x}\left(t-t_{0}-t_{\mathrm{b}}\right)^{2}, & \end{cases}
\end{aligned}
$$

where $V_{X 1}$ and $V_{X 2}$ are the vehicle longitudinal velocities with time under uniform motion and braking, respectively;
$S_{X 1}$ and $S_{X 2}$ are the vehicle longitudinal displacements with time; and $t_{\mathrm{b}}$ is the braking delay time.

3.3. Vehicle Lane-Change Model for Collision Avoidance. The safe distance is defined as the minimum distance required for the vehicle to safely change lanes or avoid a collision with the vehicle or obstacle ahead. To calculate the minimum safe distance, the critical lane changing state must be analysed based on the critical position relationship with the vehicle ahead, as shown in Figure 4.

As shown in Figure 4, the critical position relationship corresponds to the critical time $t_{\mathrm{c}}$ and the yaw angle of the host vehicle $\varphi_{0}\left(t_{c}\right)$. If the ground coordinate system $X O Y$ is rotated by $\varphi_{0}\left(t_{c}\right)$, a new coordinate system $X^{\prime} O^{\prime} Y^{\prime}$ is obtained, and the lateral displacement of the vehicle during a lane change manoeuvre on a curved section is

$$
S_{Y}\left(t_{c}\right)=S_{Y c}\left(t_{c}\right)+S_{Y 0}\left(t_{c}\right)=\frac{S_{Y c}^{\prime}\left(t_{c}\right)}{\cos \phi_{0}\left(t_{c}\right)}+S_{Y 0}\left(t_{c}\right),
$$

where

$$
\left\{S_{Y_{c}}^{\prime}\left(t_{c}\right)=\int_{0}^{t_{c}} \frac{d}{\sqrt{2 \pi} \sigma_{y}} \exp \left(\frac{-\left(t-\mu_{y}\right)^{2}}{2 \sigma_{y}^{2}}\right) \mathrm{d} t, S_{Y 0}\left(t_{\mathrm{c}}\right)=\frac{1}{R_{0}}\left(\frac{1}{4} a_{x}^{2} t_{\mathrm{c}}^{2}+\frac{2}{3} a_{x} v_{x 0} t_{\mathrm{c}}+\frac{1}{2} v_{x 0}^{2}\right) t_{\mathrm{c}}^{2},\right.
$$

where $S_{Y}\left(t_{c}\right)$ is the critical vehicle lateral displacement in $X O Y ; S_{Y 0}\left(t_{c}\right)$ and $S_{Y c}\left(t_{c}\right)$ are the critical vehicle lateral displacements for the curvature and lane-change in $X O Y$, respectively; and $S_{Y c}^{\prime}\left(t_{c}\right)$ is the vehicle lateral displacement for lane-change in $X^{\prime} O^{\prime} Y$ '.
Figure 4 shows the critical state to avoid collision and ensures that the vehicle is stable and safe during the lanechange manoeuvre. The constraint conditions of a vehicle lane-change manoeuvre for collision avoidance are

$$
\left\{\begin{array}{l}
\frac{B_{0}}{2}+\left(b+b^{\prime}\right) \sin \left(\phi_{c}\left(t_{c}\right)\right)+\frac{B_{1}}{2} \cos \left(\phi_{c}\left(t_{c}\right)\right) \leq S_{Y c}^{\prime}\left(t_{c}\right), \\
\frac{B_{0}}{2}+L \sin \left(\phi_{c}\left(t_{c}\right)\right)+B_{1} \cos \left(\phi_{c}\left(t_{c}\right)\right) \leq L_{Y c}\left(t_{c}\right)<\frac{3}{2} B, \\
a_{Y}(t)_{\max }=\frac{d \times \lambda^{2}}{\sqrt{2 \pi}\left((1 / f)+2 t_{d}\right)^{2}} \exp \left(-\frac{1}{2}\right)+a_{Y 0}(t)_{\max }, \\
a(t) \leq a_{\max }
\end{array}\right.
$$

where $B_{0}$ is the width of the front obstacle or vehicle, $B_{1}$ is the width of the host vehicle, $b$ is the distance between the centroid and the rear axle of the host vehicle, $b^{\prime}$ is the rearoverhang length of the host vehicle, $L$ is the length of the host vehicle, $\varphi_{c}\left(t_{c}\right)$ is the yaw angle of pure lane change at time $t_{\mathrm{c}}$, and $a$ and $a_{\max }$ are the acceleration and allowed maximum acceleration of the host vehicle in XOY, respectively.
3.4. Minimum Safe Distance Model. The minimum safe distance is defined as the longitudinal driving distance required to just complete obstacle avoidance. It is closely related to the relative speed and the critical time $t_{\mathrm{c}}$ and can be calculated as

$$
S_{\min }=\int_{0}^{t_{c}}\left(V_{X r}-V_{X f}\right) \mathrm{d} t+l_{s},
$$




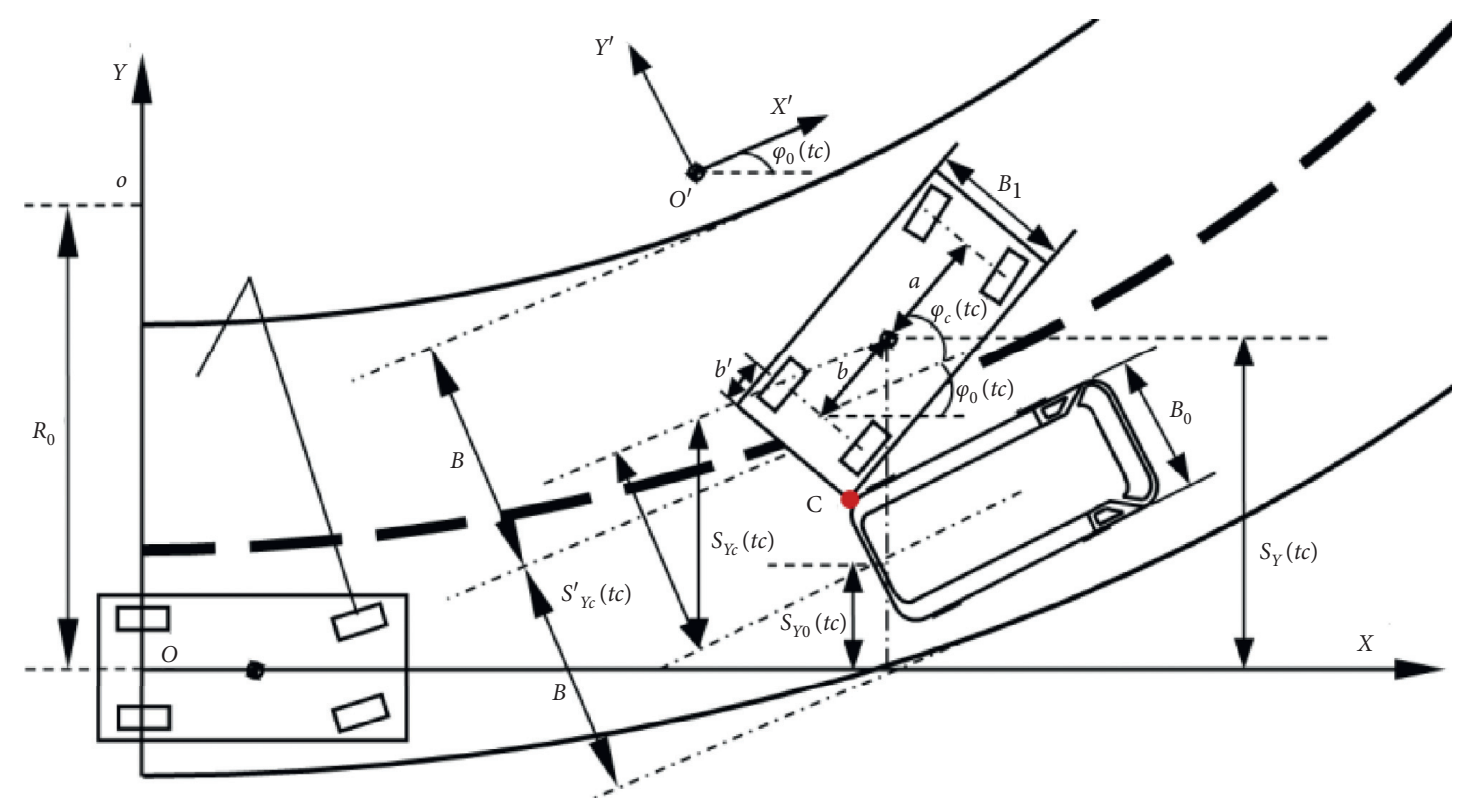

FIgURE 4: Critical position relationship for safe lane change.

where $S_{\min }$ is the minimum safe distance; $l_{\mathrm{s}}$ is the conservative safe clearance, set as $5 \mathrm{~m}$ herein; and $\Delta V_{X}$ is the relative longitudinal velocity between the host vehicle and the obstacle ahead.

The relative speed can be obtained using GPS or sensors, and consequently, the key to calculate the minimum safety distance is to determine $t_{\mathrm{c}}$. According to equation (11), $t_{\mathrm{c}}$ is determined by the minimum safe lateral displacement $S_{Y c}^{\prime}\left(t_{\mathrm{c}}\right)_{\min }$. As $\left(b+b^{\prime}\right)>B_{1} / 2$, in general, $S_{Y \mathrm{c}}^{\prime}\left(t_{\mathrm{c}}\right)_{\min }$ is proportional to the maximum yaw angle of the host vehicle during lane change $\varphi_{\mathrm{c}}(t)_{\max }$ and can be conservatively calculated as

$S_{Y_{c}}^{\prime}\left(t_{c}\right)_{\min }=\frac{B_{0}}{2}+\left(b+b^{\prime}\right) \sin \left(\phi_{c}(t)_{\max }\right)+\frac{B_{1}}{2} \cos \left(\phi_{c}(t)_{\max }\right)$,

where $\varphi_{\mathrm{c}}\left(t_{\mathrm{c}}\right)_{\max }$ can be estimated as

$$
\phi_{\mathrm{c}}(t)_{\max }=\left[V_{Y \mathrm{c}}(t) / V_{X \mathrm{c}}(t)\right]_{\max } .
$$

According to equation (6), when $t=\mu_{y}$, the vehicle lateral velocity is maximum $V_{Y c}(t)_{\max }$. If the vehicle is in the critical position required for collision avoidance at this moment, $\varphi_{c}(t)_{\max }$ can be calculated and $S_{Y_{c}}^{\prime}\left(t_{c}\right)_{\min }$ can be obtained. Subsequently, $t_{c}$ can be obtained through backward deduction, based on the vehicle lane-change trajectory.

3.5. Model Validation. The proposed model was validated using the autopilot mode of a physics-based simulation platform called PreScan, developed by Siemens, Germany. As an active safety experimental platform, PreScan can build scenes based on the actual road environment and sensor model and is an advanced and professional development tool. Therefore, it is widely used in the automotive industry to develop Advanced Driver Assistance Systems (ADASs) and autonomous vehicles.
Using PreScan, a vehicle lane-change scenario for collision avoidance was simulated, considering a two-way fourlane highway with a curved section and moderate rainfall. The vehicle and road parameters were set up as listed in Table 1. Subsequently, critical samples were selected to obtain the lane-change paths, considering a steering frequency of $0.4 \mathrm{~Hz}$ and a decision making and execution time of $0.5 \mathrm{~s}$. The minimum safety distances were calculated at a constant speed and braking deceleration $\left(2 \mathrm{~m} / \mathrm{s}^{2}\right)$. Comparative verifications are shown in Figure 5.

As shown in Figure 5, the trends of the vehicle motions and trajectories obtained using PreScan and the proposed lane-change model are consistent. Related parameters such as the maximum vehicle lateral acceleration, yaw angle, critical time point for collision avoidance, minimum safety distance, and mean square errors between the vehicle paths were calculated as well and are listed in Table 2 .

As shown in Figure 5 and Table 2, the trends of the vehicle lateral velocities and trajectories obtained using the proposed model are in good agreement with the PreScan results. Specifically, the errors of the typical dynamic parameters, including the maximum vehicle lateral acceleration, are less than $5 \%$ and are fairly consistent. Moreover, the maximum vehicle lateral acceleration determined by the lane-change model is about $3.5 \mathrm{~m} / \mathrm{s}^{2}$, which is much lower than the critical value of road adhesion (about $5.2 \mathrm{~m} / \mathrm{s}^{2}$ ). Thus, the lane-change model can accurately describe the trajectory and dynamics of a lane-change manoeuvre on a curved road section and effectively express the vehicle handling and lateral motion characteristics through a simple fitting process.

\section{Collision Avoidance Decision and Control}

4.1. Decision Mechanism for Collision Avoidance. An autonomous vehicle must be able to make the right decision 
TABLE 1: Basic parameters of the simulation vehicle and road.

\begin{tabular}{lcccc}
\hline Road width $(\mathrm{m})$ & Road adhesion coefficient & Curve radius $(\mathrm{m})$ & Initial velocity $(\mathrm{m} / \mathrm{s})$ & Vehicle width $(\mathrm{m})$ \\
\hline 3.75 & 0.53 & 650 & 30 & 2 \\
\hline
\end{tabular}
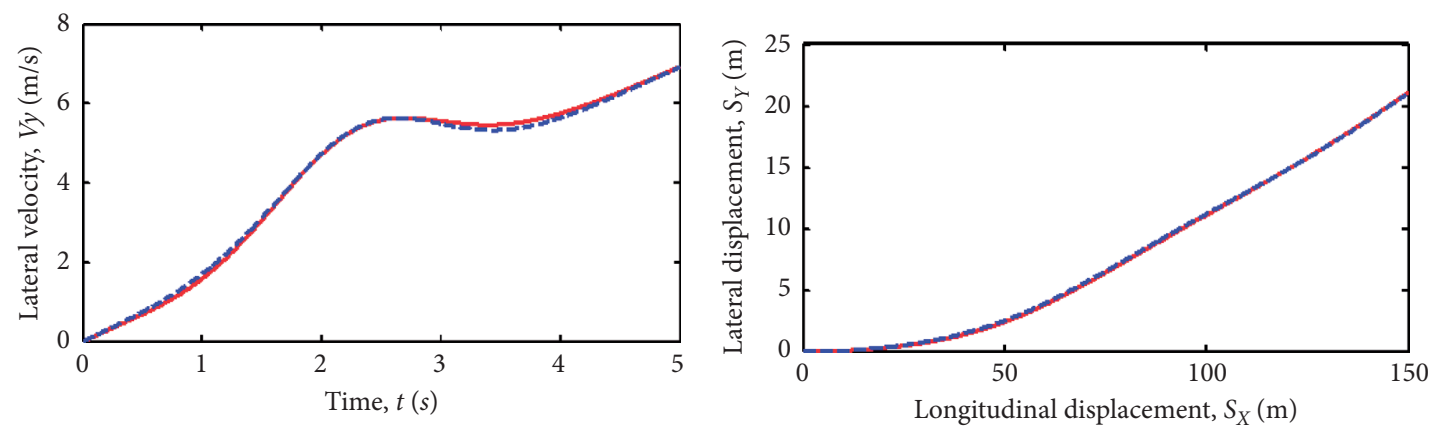

- PreScan

- - - Lane-change model
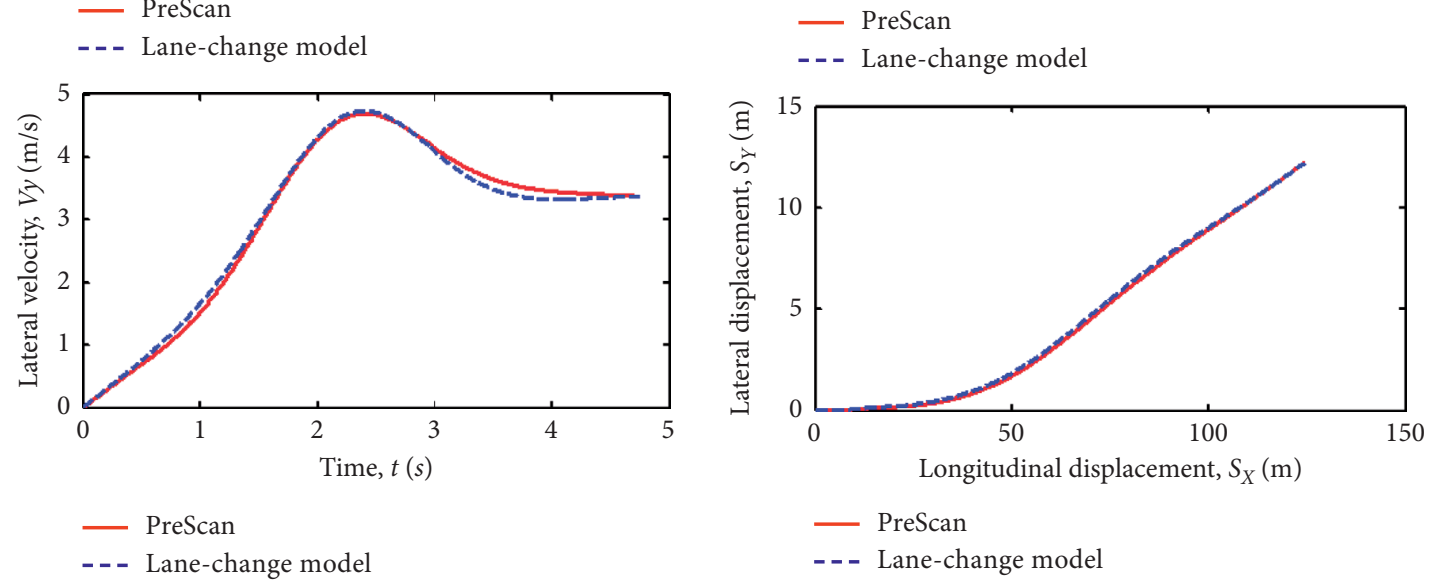

(a)

(b)

Figure 5: Comparative verifications with different simulation tools. (a) Lateral velocity at constant speed and $0.4 \mathrm{~Hz}$ steering. (b) Global trajectory at constant speed and $0.4 \mathrm{~Hz}$ steering.

TABLE 2: Detailed comparison by different simulation tools.

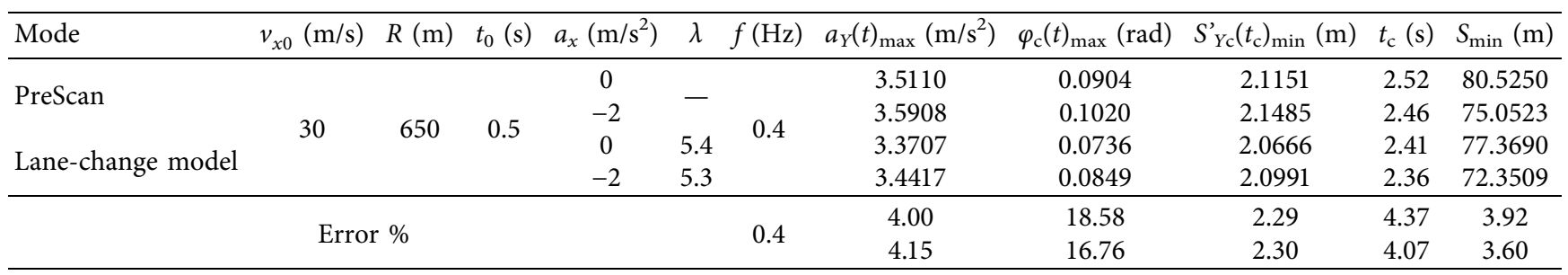

and plan a reasonable path according to the external situation detected by the perception sensors on the vehicle, such as radar, CCD, and inertial gyroscopes. Consequently, the decision mechanism must generate an appropriate path and provide the basis for the control inputs, making it an important part of an autonomous driving system. Considering driving safety, comfort, and efficiency, we propose a scoring reference-based lane-change decision mechanism for collision avoidance on a curved road with low adhesion and formulate a four-mode decision-making rule based on the safe lane-change model.
Considering a relative velocity of $30 \mathrm{~m} / \mathrm{s}$ between the autonomous vehicle and the vehicle (or obstacle) ahead and using simple driving modes (e.g., constant speed and braking with a deceleration of $2 \mathrm{~m} / \mathrm{s}^{2}$ ), we proposed decision-making scoring references, as listed in Table 3.

Table 3 shows the four scoring reference hierarchies for decision-making. The first is safety, which is the most important consideration for determining the feasibility of the drive mode. It is estimated based on the longitudinal headway and the lateral acceleration. If the difference between the current headway and the minimum safe headway 
TABLE 3: Hierarchical scoring reference with relative velocity of $30 \mathrm{~m} / \mathrm{s}$.

\begin{tabular}{|c|c|c|c|c|c|c|}
\hline Hierarchy & Performance & $\begin{array}{l}\text { Driving mode steering } \\
\text { frequency }(\mathrm{Hz}) / \mathrm{brake} \\
\text { deceleration }\left(\mathrm{m} / \mathrm{s}^{2}\right)\end{array}$ & $\begin{array}{c}\text { Maximum } \\
\text { acceleration, } \\
a(t)_{\max }\left(\mathrm{m} / \mathrm{s}^{2}\right)\end{array}$ & $\begin{array}{l}\text { Minimum safe } \\
\text { headway, } S_{\text {min }} \\
(\mathrm{m})\end{array}$ & Evaluation parameter & Score \\
\hline 1 & Overall & - & - & - & - & $s_{1}=s_{2} \times\left(i \times s_{3}+j \times s_{4}\right)$ \\
\hline 2 & Safety & - & $\leq a_{Y \max }$ & $\Delta L \geq S_{\min }$ & $\begin{array}{c}\Delta: \text { difference between } \\
\text { headway and } \\
\text { minimum safe } \\
\text { headway }\end{array}$ & $\begin{array}{l}s_{2}=1(\Delta>0) \\
s_{2}=0(\Delta \leq 0)\end{array}$ \\
\hline \multirow{10}{*}{3} & \multirow{10}{*}{ Comfort } & $0.1 / 0$ & 1.5973 & 192.5480 & \multirow{10}{*}{$\begin{array}{c}\text { Maximum } \\
\text { acceleration }\end{array}$} & $s_{3}=10$ \\
\hline & & $0.2 / 0$ & 2.0718 & 117.2420 & & $s_{3}=9$ \\
\hline & & $0.1 / 2$ & 2.5900 & 146.2759 & & $s_{3}=8$ \\
\hline & & $0.3 / 0$ & 2.7085 & 90.8600 & & $s_{3}=7$ \\
\hline & & $0.2 / 2$ & 2.9122 & 102.3528 & & $s_{3}=6$ \\
\hline & & $0.4 / 0$ & 3.3707 & 77.3690 & & $s_{3}=5$ \\
\hline & & $0.3 / 2$ & 3.3839 & 83.1736 & & $s_{3}=4$ \\
\hline & & $0.4 / 2$ & 3.9806 & 72.1616 & & $s_{3}=3$ \\
\hline & & $0.5 / 0$ & 4.2781 & 68.8610 & & $s_{3}=2$ \\
\hline & & $0.5 / 2$ & 4.8007 & 65.1585 & & $s_{3}=1$ \\
\hline \multirow{10}{*}{4} & \multirow{10}{*}{ Efficiency } & $0.5 / 2$ & 4.8007 & 65.1585 & \multirow{10}{*}{$\begin{array}{l}\text { Minimum safe } \\
\text { headway }\end{array}$} & $s_{4}=10$ \\
\hline & & $0.5 / 0$ & 4.2781 & 68.8610 & & $s_{4}=9$ \\
\hline & & $0.4 / 2$ & 3.9806 & 72.1616 & & $s_{4}=8$ \\
\hline & & $0.4 / 0$ & 3.3707 & 77.3690 & & $s_{4}=7$ \\
\hline & & $0.3 / 2$ & 3.3839 & 83.1736 & & $s_{4}=6$ \\
\hline & & $0.3 / 0$ & 2.7085 & 90.8600 & & $s_{4}=5$ \\
\hline & & $0.2 / 2$ & 2.9122 & 102.3528 & & $s_{4}=4$ \\
\hline & & $0.2 / 0$ & 2.0718 & 117.2420 & & $s_{4}=3$ \\
\hline & & $0.1 / 2$ & 2.5900 & 146.2759 & & $s_{4}=2$ \\
\hline & & $0.1 / 0$ & 1.5973 & 192.5480 & & $s_{4}=1$ \\
\hline
\end{tabular}

is above zero, the lateral acceleration is less than the threshold and the manoeuvre is safe to carry out; if not, it is dangerous. The second scoring reference is comfort, which is determined by the acceleration of the vehicle; the smaller the acceleration, the better the comfort, in principle. The third is the efficiency; the smaller the minimum safe headway, the higher the efficiency of changing lanes to avoid a collision. It should be emphasised that even though the relative velocity is assumed to be $30 \mathrm{~m} / \mathrm{s}$, it does not affect the decisionmaking mechanism of lane changing, nothing but the specific safe distance and lateral acceleration change with the change in velocity. The overall decision-making score is

$$
s_{1}=s_{2} \times\left(i \times s_{3}+j \times s_{4}\right),
$$

where $s_{1}$ is the overall score of the driving mode; $s_{2}, s_{3}$, and $s_{4}$ are the safety, comfort, and efficiency scores, respectively; and $i$ and $j$ are the weight coefficients for comfort and efficiency, respectively. In this study, $i=0.9$ and $j=0.1$, which indicates a much higher emphasis on comfort.

Based on the scoring references under specific circumstances, a four-level lane-change mode for collision avoidance is proposed, as listed in Table 4.

Table 4 demonstrates the intelligent decision making and the four-level lane-change mode generation processes, based on the scoring method. First, the application conditions are defined by the vehicle relationship relative to the typical parameters, such as headway and relative speed. Second, using the lane-change model, procedures are obtained based on the minimum safe headway and maximum acceleration.
Third, the optimal procedures for different application conditions are determined based on the highest score. The basic principle is that safety always comes first, even if comfort and efficiency are low. The weights of comfort and efficiency have a significant influence on the optimal procedures. Therefore, the intelligent decision-making mechanism can be applied to different driving styles, including those of robust and radical drivers.

4.2. Vehicle Control Model. Model predictive control (MPC) was developed in the late 1970s and includes methods such as dynamic matrix control (DMC) and model algorithmic control (MAC). It is a well-known and effective control algorithm and has found widespread acceptance in industry. The major advantage of MPC is its ability to handle multivariable interactions and operating constraints in a systematic manner. Essentially, it is a type of optimisation method based on objective functions, and rolling horizon optimal control is achieved by model-based state prediction, optimisation with constraints in a finite time domain, and feedback correction. This section presents a detailed MPC controller derived from a nonlinear four-degree-of-freedom (4-DOF) vehicle model that can simultaneously track the reference planned path and velocity.

Considering the longitudinal and lateral coupling movement of a vehicle during typical steering dynamics, we established a 4-DOF vehicle model based on reasonable simplification, involving front wheel steering and vehicle longitudinal, lateral, and yaw motions: 
TABle 4: Four-level lane-change mode for collision avoidance.

\begin{tabular}{|c|c|c|c|c|c|}
\hline Mode & $\begin{array}{l}\text { Host vehicle } \\
\text { initial velocity } \\
\qquad(\mathrm{m} / \mathrm{s})\end{array}$ & Application condition & $\begin{array}{l}\text { Optional driving mode steering } \\
\text { frequency }(\mathrm{Hz}) / \text { brake deceleration } \\
\qquad\left(\mathrm{m} / \mathrm{s}^{2}\right)\end{array}$ & Score & $\begin{array}{l}\text { Optimal driving mode steering } \\
\text { frequency }(\mathrm{Hz}) / \text { brake deceleration } \\
\qquad\left(\mathrm{m} / \mathrm{s}^{2}\right)\end{array}$ \\
\hline \multirow{8}{*}{1} & \multirow{15}{*}{30} & \multirow{8}{*}{$\Delta L+\int_{0}^{t_{\mathrm{c}}} V_{X \mathrm{f}} \mathrm{d} t+l_{\mathrm{s}} \geq 120$} & $0.2 / 0$ & 8.4 & \multirow{8}{*}{$0.2 / 0$} \\
\hline & & & $0.3 / 0$ & 6.8 & \\
\hline & & & $0.2 / 2$ & 5.8 & \\
\hline & & & $0.4 / 0$ & 5.2 & \\
\hline & & & $0.3 / 2$ & 4.2 & \\
\hline & & & $0.4 / 2$ & 3.5 & \\
\hline & & & $0.5 / 0$ & 2.7 & \\
\hline & & & $0.5 / 2$ & 1.9 & \\
\hline \multirow{7}{*}{2} & & \multirow{7}{*}{$100 \leq \Delta L+\int_{0}^{t_{\mathrm{c}}} V_{X \mathrm{f}} \mathrm{d} t+l_{\mathrm{s}}<120$} & $0.3 / 0$ & 6.8 & \multirow{7}{*}{$0.3 / 0$} \\
\hline & & & $0.2 / 2$ & 5.8 & \\
\hline & & & $0.4 / 0$ & 5.2 & \\
\hline & & & $0.3 / 2$ & 4.2 & \\
\hline & & & $0.4 / 2$ & 3.5 & \\
\hline & & & $0.5 / 0$ & 2.7 & \\
\hline & & & $0.5 / 2$ & 1.9 & \\
\hline \multirow{5}{*}{3} & & \multirow{5}{*}{$80 \leq \Delta L+\int_{0}^{t_{\mathrm{c}}} V_{X \mathrm{f}} \mathrm{d} t+l_{\mathrm{s}}<100$} & $0.4 / 0$ & 5.2 & \multirow{5}{*}{$0.4 / 0$} \\
\hline & & & $0.3 / 2$ & 4.2 & \\
\hline & & & $0.4 / 2$ & 3.5 & \\
\hline & & & $0.5 / 0$ & 2.7 & \\
\hline & & & $0.5 / 2$ & 1.9 & \\
\hline 4 & & $66 \leq \Delta L+\int_{0}^{t_{\mathrm{c}}} V_{X \mathrm{f}} \mathrm{d} t+l_{\mathrm{s}}<80$ & $0.5 / 2$ & 1.9 & $0.5 / 2$ \\
\hline
\end{tabular}

$$
\left\{\begin{array}{l}
\dot{X}=f(\mathbf{X})+g(\mathbf{X}) \mathbf{u}, \\
\mathbf{X}=\left[x_{1}, x_{2}, x_{3}, x_{4}, x_{5}, x_{6}\right]^{T} \\
=\left[V_{X}, V_{Y}, \dot{\phi}, \phi, \dot{\delta}_{\mathrm{f}}, \delta_{\mathrm{f}}\right]^{T},
\end{array}\right.
$$

where $V_{X}$ and $V_{Y}$ are the longitudinal and lateral velocities, respectively; $\varphi$ is the yaw angle; and $\delta_{\mathrm{f}}$ is the front wheel steering angle.

Setting $V_{X}, V_{Y \mathrm{t}}, \varphi$, and $\delta_{\mathrm{f}}$ as the control output variables and applying the typical control strategies of active front steering (AFS) and direct yaw control (DYC), the vehicle control model can be expressed as

$$
\begin{aligned}
&\left\{\begin{array}{l}
\dot{X}=f(\mathbf{X})+g(\mathbf{X}) \mathbf{u}, \\
\mathbf{Z}=\left[\begin{array}{llll}
V_{X} & V_{Y} & \phi & \delta_{\mathrm{f}}
\end{array}\right]^{T},
\end{array}\right. \\
& \mathbf{u}=\left[\begin{array}{lllll}
u_{1} & u_{2} & u_{3} & u_{4}
\end{array}\right]\left[\begin{array}{llll}
F_{x \mathrm{fc}} & F_{x \mathrm{rc}} & M_{\mathrm{c}} & \delta_{\mathrm{fc}}
\end{array}\right] .
\end{aligned}
$$

The function of a controller is to guarantee tracking and robust stability considering the nonlinear effect of the vehicle.

For an MPC controller, linearisation and discretisation can be used to improve the real-time performance of the controller. The nonlinear vehicle dynamic model can be linearised as a linear time invariant (LTI) state space form, using the first order approximation of the Taylor expansion. Subsequently, the linearised model can be discretised as

$$
\begin{aligned}
& \left\{\begin{array}{l}
\dot{X}=A_{j} \mathbf{X}(t)+B_{j} \mathbf{u}(t), \\
\mathbf{X}(k+1)=A_{k} \mathbf{X}(k)+B_{k} \mathbf{u}(k), \\
\mathbf{H}(k)=C_{k} \mathbf{G}(k)+D_{k} \Delta \mathbf{U}(k),
\end{array}\right. \\
& \left\{\begin{array}{l}
A_{k}=1+T A_{j}, \\
B_{k}=T B_{j},
\end{array}\right.
\end{aligned}
$$

where

$$
\mathbf{H}(k)=\left[\begin{array}{c}
\mathbf{Z}(k+1 \mid k) \\
\mathbf{Z}(k+2 \mid k) \\
\vdots \\
\mathbf{Z}\left(k+N_{c} \mid k\right) \\
\vdots \\
\mathbf{Z}\left(k+N_{p} \mid k\right)
\end{array}\right],
$$




$$
\begin{aligned}
& C(k)=\left[\begin{array}{c}
C_{k, k} A_{k, k} \\
C_{k, k} A_{k, k} A_{k+1, k} \\
\vdots \\
C_{k, k} \prod_{i=k}^{k+N_{c}} A_{k, k} \\
\vdots \\
C_{k, k} \prod_{i=k}^{k+N_{p}-1} A_{k, k}
\end{array}\right] \\
& D(k)=\left[\begin{array}{cccc}
C_{k, k} B_{k, k} & 0 & 0 & 0 \\
C_{k, k} A_{k, k} B_{k, k} & C_{k, k} B_{k, k} & 0 & 0 \\
\ldots & \ldots & \ddots & \ldots \\
C_{k, k} \prod_{i=k}^{k+N_{c}} A_{k, k} B_{k, k} & C_{k, k} \prod_{i=k}^{k+N_{c}-1} A_{k, k} B_{k, k} & \ldots & C_{k, k} B_{k, k} \\
\vdots & \vdots & \cdots & \vdots \\
C_{k, k} \prod_{i=k}^{k+N_{p}-1} A_{k, k} B_{k, k} & C_{k, k} \prod_{i=k}^{k+N_{p}-2} A_{k, k} B_{k, k} & \ldots & C_{k, k} \\
\prod_{i=k}^{k+N_{p}-N_{c}-1}
\end{array}\right. \\
& G(k)=\left[\begin{array}{cc}
\mathbf{X}(k \mid & k) \\
\mathbf{u}(k-1 \mid k) &
\end{array}\right] \text {; } \\
& \Delta \mathbf{U}(k)=\left[\begin{array}{c}
\Delta \mathbf{u}(k+1 \mid k) \\
\Delta \mathbf{u}(k+2 \mid k) \\
\vdots \\
\Delta \mathbf{u}\left(k+N_{c} \mid k\right) \\
\vdots \\
\Delta \mathbf{u}\left(k+N_{p} \mid k\right)
\end{array}\right],
\end{aligned}
$$

where, $A_{j}, B_{j}, A_{k}, B_{k}, C_{k}$, and $D_{k}$ are the Jacobian matrices at the working point; $T$ is the sample time; and $N_{\mathrm{p}}$ and $N_{\mathrm{c}}$ are the predictive horizon and control horizon, respectively.

While designing the controller, constraints must be set to ensure that the autonomous vehicle can precisely and stably track the trajectory references generated during intelligent path planning. The constraints designed herein primarily include two aspects-one related to the vehicle control quantity and the other related to the vehicle state quantity:

$$
\left\{\begin{array}{l}
\mathbf{U}_{\min } \leq \mathbf{U} \leq \mathbf{U}_{\max } \\
\sqrt{a_{X}^{2}+a_{Y}^{2}} \leq \mu g .
\end{array}\right.
$$

In addition to tracking the reference precisely and stably, the controller must also keep the vehicle running at the desired speed. The cost quadratic function is

$$
\begin{aligned}
J= & \sum_{i=1}^{N_{p}}\left\|\mathbf{H}_{\mathrm{ref}}(k+i \mid k)-\mathbf{H}(k+i \mid k)\right\|_{\mathbf{Q}}^{2}+\sum_{i=0}^{N_{c}-1}\|\Delta \mathbf{U}(k+i \mid k)\|_{\mathbf{R}}^{2} \\
& +\sum_{i=0}^{N_{c}-1}\|\mathbf{U}(k+i \mid k)\|_{\mathbf{S}}^{2}+\boldsymbol{\rho} \varepsilon^{2},
\end{aligned}
$$

where $\mathbf{H}_{\text {ref }}$ is a matrix of the reference outputs and $\mathbf{Q}, \mathbf{R}, \mathbf{S}$, and $\boldsymbol{\rho}$ are the weight matrices for the system outputs $H(k)$, the control increment $\Delta U(k)$, the control inputs $U(k)$, and the relaxation factor $\varepsilon$.

According to [38], the optimal problem for MPC can be converted to a standard quadratic programming problem. The decision variable is denoted by $\mathbf{U}$, and the objective function $J$ and the constraints can be expressed as

$$
J_{\min }=\frac{1}{2} \Delta \mathbf{U}^{T} \mathbf{M} \Delta \mathbf{U}+\mathbf{G}^{T} \Delta \mathbf{U}
$$

where $\mathbf{M}$ and $\mathbf{G}$ are compatible matrices and vectors in the quadratic programming problem. The incremental control inputs $\Delta \mathbf{U}$ of the constraints are

$$
\Delta \mathbf{U}_{\min } \leq \Delta \mathbf{U} \leq \Delta \mathbf{U}_{\max }
$$

The solution of the quadratic program problem can be solved using quadprog in MATLAB, and the activeset algorithm was chosen herein.

Figure 6 shows the structure of the safe lane-change intelligent control system. Initially, the environment and ambient obstacles are detected by on-board sensors (radar, cameras, etc.). When obstacles are detected, specific 
information, such as the relative speed and headway, is set as the input of the autonomous vehicle decision mechanism. Feasible path planning and trajectory references (including path and speed) are generated based on the decision mechanism. Subsequently, using the linearised MPC algorithm and the AFS and DYC strategies, the designed controller determines the vehicle control inputs though prediction and convex optimisation, to ensure that the vehicle follows the planned trajectory.

4.3. Control Performance Validation. Two typical scenarios were defined to verify the performance of the designed controller. The first is a simple lane-change scenario to evaluate the controller against other conventional methods, such as single preview and PID. The second scenario is a continuous curve with multiple lane-changes. The details of the two scenarios are as follows:

(1) An autonomous vehicle travelling at $20 \mathrm{~m} / \mathrm{s}$, with a steering frequency of $0.2 \mathrm{~Hz}$ and a decision time of $1.5 \mathrm{~s}$, on a straight highway.

(2) An autonomous vehicle travelling at $30 \mathrm{~m} / \mathrm{s}$, with a steering frequency of $0.4 \mathrm{~Hz}$ and a distance of $80 \mathrm{~m}$ to avoid collision with obstacles, on a continuous curved road composed of three parts with circular arcs of $600 \mathrm{~m}$ radii.

The control performance of the first scenario was verified by comparing the proposed controller with conventional control algorithms, such as a PID controller and a controller without path planning, as shown in Figure 7.

Figure 7 shows the control results of different models. The proposed MPC controller has a better control effect than the other control models. Figures $7(a)$ and $7(b)$ indicate that the proposed controller has better tracking, and the lateral error is around $0.02 \mathrm{~m}$, which is better than that of the PID controller $(0.05 \mathrm{~m})$ and that of the model without path planning $(0.4 \mathrm{~m})$. Figure $7(\mathrm{c})$ demonstrates that the lateral velocity is precisely controlled, not only in terms of the change tren, but also considering the value and real time performance; the error is about $1 \%$, while that of the PID is about $5 \%$, with a delay of about $0.1 \mathrm{~s}$. Figure 7 (d) illustrates the yaw motion, and the proposed controller shows similar characteristics, while the PID generates a larger error and delay. Thus, the proposed MPC controller based on the 4DOF model offers better tracking and robust stability.

The multiple decision-making and anticollision control performance of the MPC controller were verified based on the second scenario. The simulation results are shown in Figure 8.

Figure $8(a)$ and $8(\mathrm{~b})$ illustrate the global continuous overtaking trajectory and the tracking error of the autonomous vehicle; the absolute error is less than $0.04 \mathrm{~m}$. Although the error of the combined procedure with lane changing and turning exceeds $0.02 \mathrm{~m}$, it still provides a good tracking effect relative to the desired reference performance. As shown in Figure 8(c) and 8(d), the longitudinal and lateral motions of the vehicle are decoupled and controlled reasonably well, despite small-amplitude longitudinal fluctuations that are slightly larger than the reference. The lateral velocity is in a continuous large-scale form but is well controlled. Figure 8(e) and 8(f) demonstrate that the second-order dynamics also have good consistency with the desired references, and the absolute error is less than $4 \%$ with a delay of about $0.1 \mathrm{~s}$. This indicates that the vehicle is well controlled by the AFS and DYC. As shown in Figure $8(\mathrm{~g})$ and $8(\mathrm{~h})$, the control inputs are continuous, and the changing trend of the steering input is consistent with the vehicle dynamics; the delay time is about $0.5 \mathrm{~s}$. Furthermore, the vehicle lateral acceleration is less than $4.6 \mathrm{~m} /$ $\mathrm{s}^{2}$, and the absolute longitudinal forces of the tractor and semitrailer axles are below $1000 \mathrm{~N}$, which is considerably lower than the saturation forces of the tyres, even with the low coefficient of adhesion due to rainfall. Therefore, the vehicle is controlled with absolute stability during lanechanging and cornering on a highway in rainy weather, and the proposed controller is capable of obstacle avoidance on a curved highway section.

\section{Discussion and Conclusion}

Based on the vehicle steering and lateral dynamics in rainy weather, we introduced Gaussian distribution to the lateral dynamics of an autonomous vehicle for decision making and path planning. In particular, the scenario of an autonomous vehicle travelling on a curved highway section in rainy weather was considered herein. The main conclusions are

(1) The Gaussian distribution function could perfectly describe the lateral dynamics of the vehicle. The related parameters were easily fitted, and the characteristics of autonomous vehicles, including reaction, response, pavement condition, and lane-change status, were accurately and quantitatively obtained. The reaction time of decision-making and path planning, steering frequency, delay times of vehicle lateral dynamics, road adhesion coefficient, and completion probability of lane change were determined as well. In a typical scenario, the proposed lane-change model could be applied to the path planning process of a vehicle lane-changing manoeuvre.

(2) Based on the lane-change model, the minimum safe distance for collision avoidance was obtained. The lane-change decision mechanism utilises a scoring system that considers driving safety, comfort, and efficiency and serves as a reference for intelligent path planning.

(3) Using convex optimisation, an MPC controller with AFS and DYC was designed and verified. The results of the typical scenarios considering an autonomous vehicle in rainy weather demonstrated that the proposed controller has good tracking and robust stability. This study offers practical value as it considers the cooperative relationship between humans, vehicles, the road, and the environment and can serve as valuable reference in the development of autonomous driving systems. 


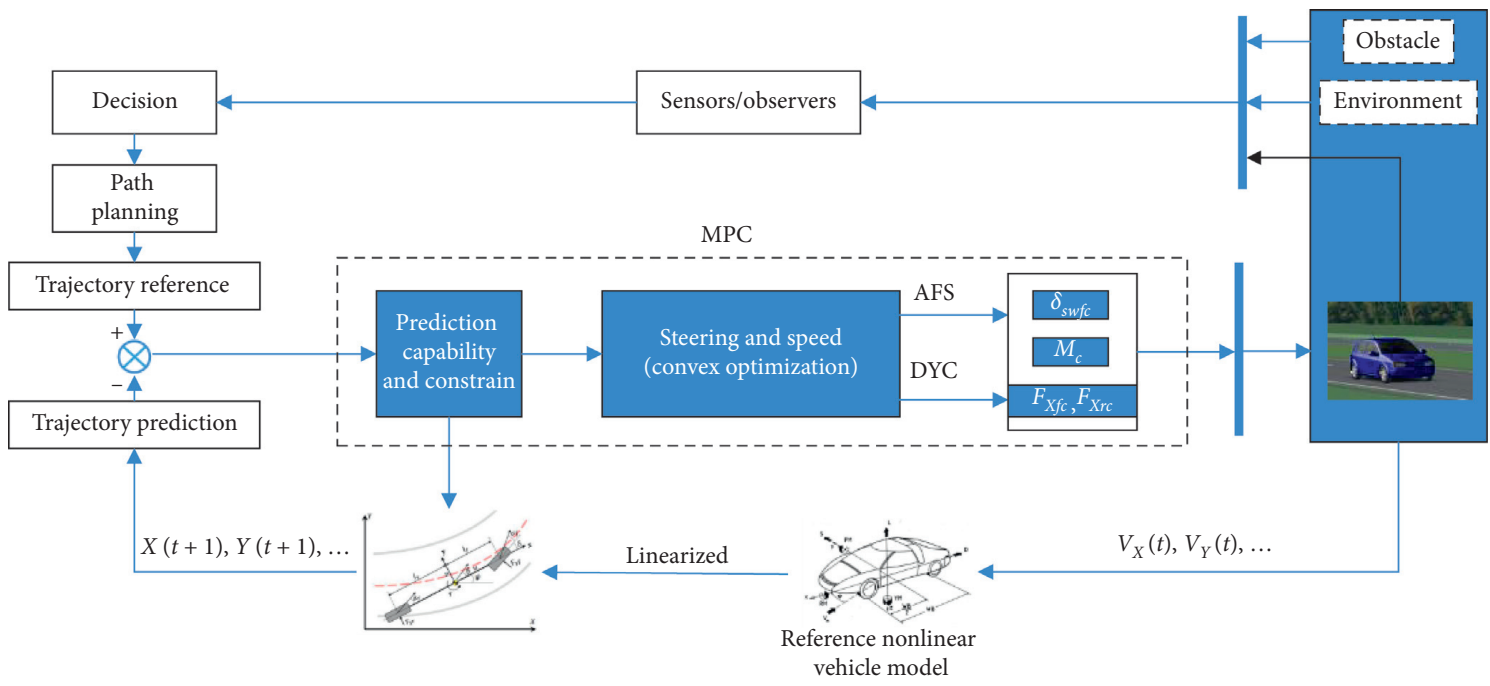

FIGURE 6: Structure of the safe lane-change intelligent control system.

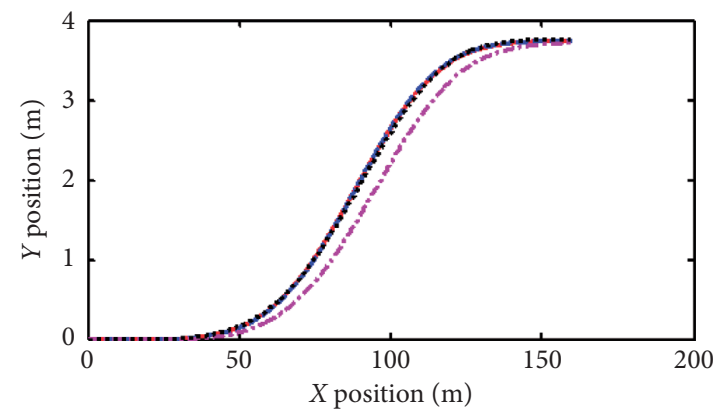

- Reference - - - MPC

$\cdot-\cdot-$ Single point preview ..... PID

(a)

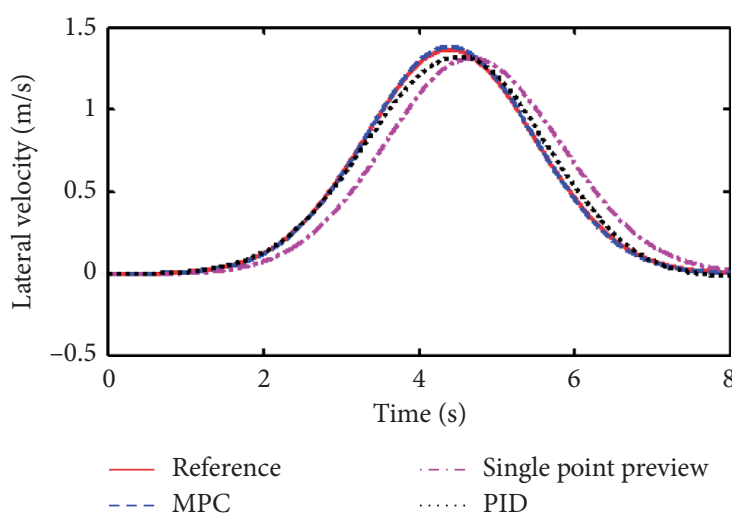

(c)

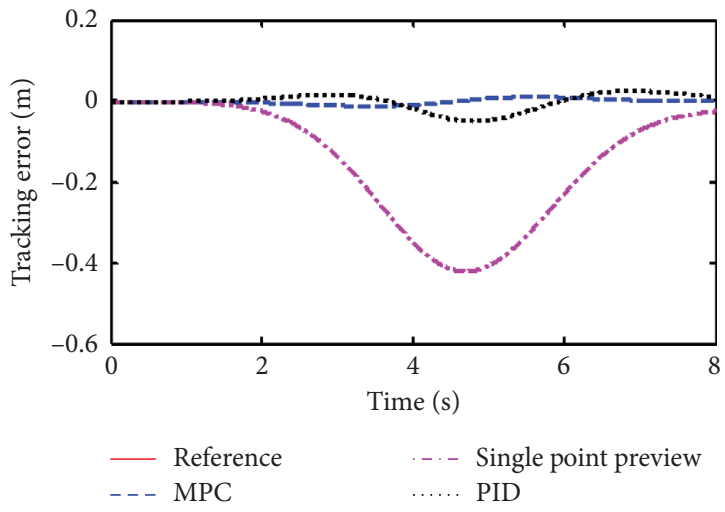

(b)

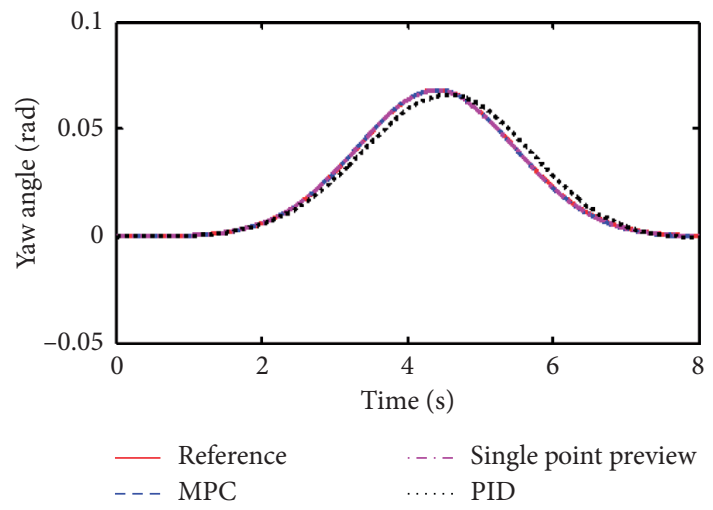

(d)

Figure 7: Model comparative study of controller. (a) Global path. (b) Tracking error. (c) Lateral velocity. (d) Yaw angle.

The results obtained herein are of significant importance for numerous applications and provide a valuable reference for future studies involving actual vehicles. Compared to traditional studies on lane-change manoeuvres, the novel trajectory model, decision mechanism, and control algorithm proposed herein can describe the system characteristics under various road alignments and operational environments. However, considering the complex environmental influences on man-machine interactions, further research is required. The multifactor coupling characteristics 

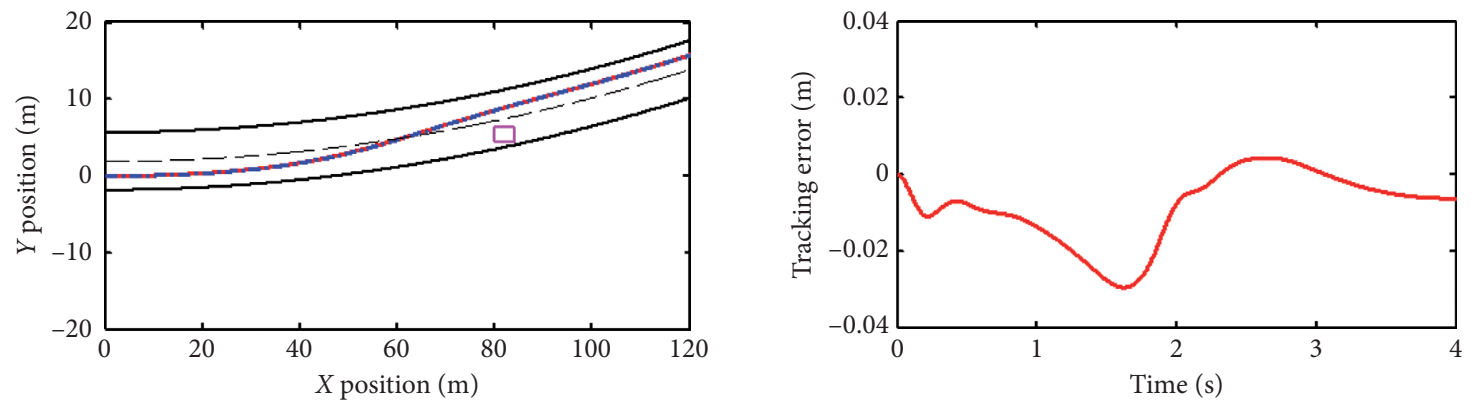

- Reference

- - - Control model

ㅁ Obstacle

(a)

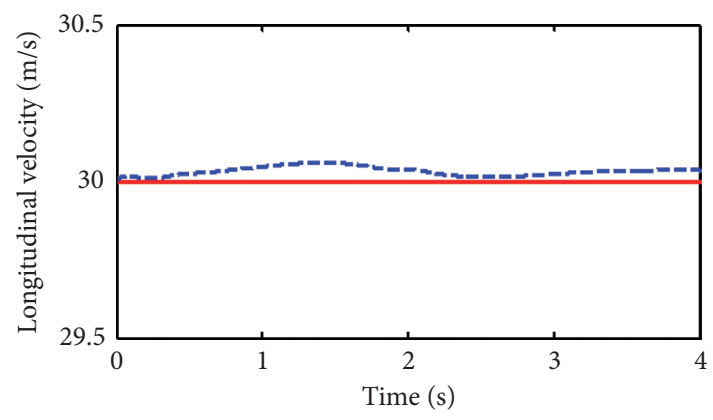

- Reference

- - - Control model

(c)

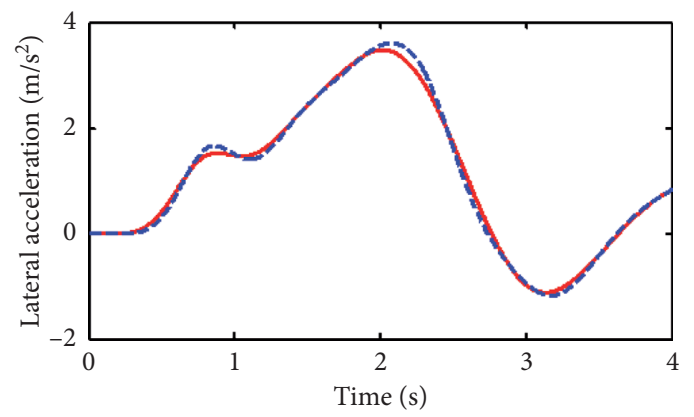

- Reference

- - - Control model

(e)

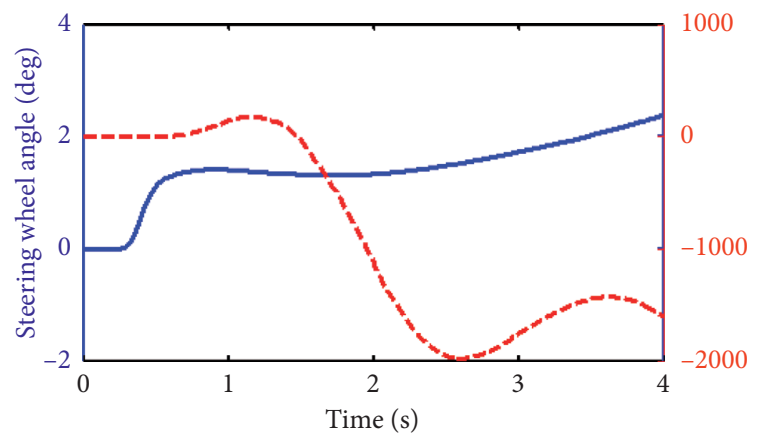

(g) (b)

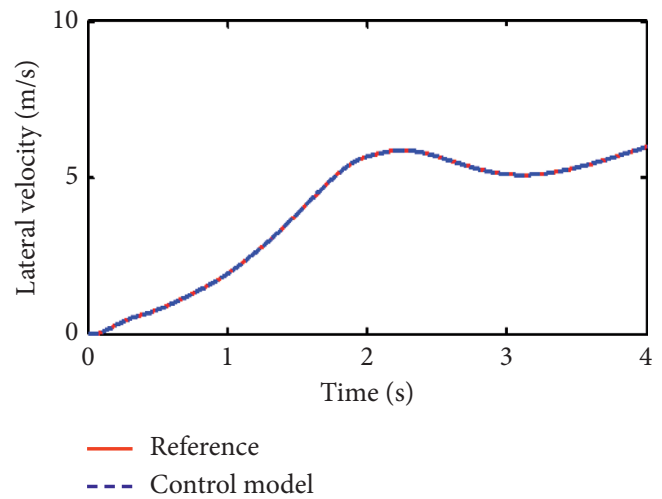

(d)

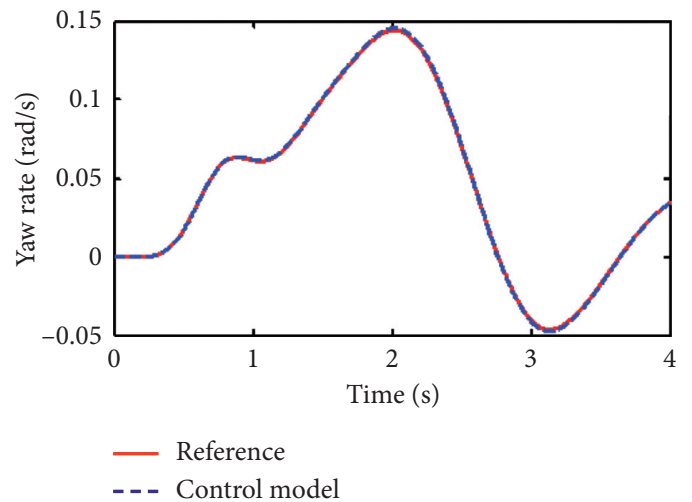

(f)

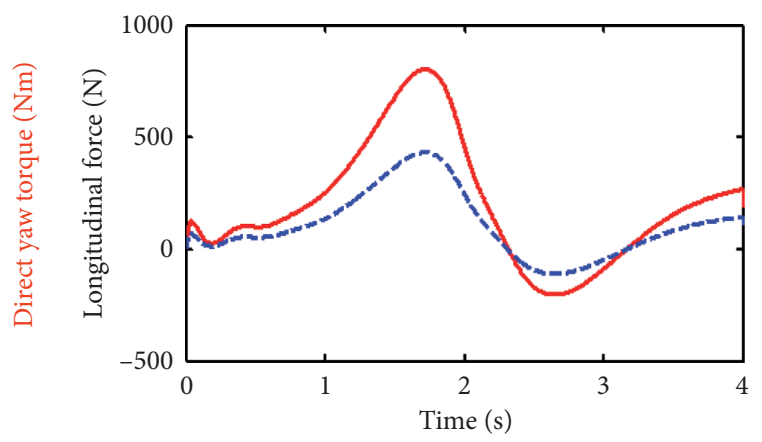

- Front axle

(g) (h)

Figure 8: Control effects of specific modes for anticollision on a curved road. (a) Global path. (b) Tracking error. (c) Longitudinal velocity. (d) Lateral velocity. (e) Lateral acceleration. (f) Yaw rate. (g) Control inputs of AFS and DYC. (h) Control inputs of longitudinal forces. 
of the environmental impact must be studied further. Typical examples include the interference between the perception sensors on the vehicle that detect the external environment. Notably, human driving behaviour always forms the basis for autonomous vehicle development. Consequently, a systematic study of personified decisionmaking is required to ensure coordination between humans and the environment. Furthermore, considering the field of cooperative vehicle-infrastructure systems, a cooperative decision-making and control strategy with swarm intelligence for multiple vehicles or vehicle fleets should be studied in depth.

\section{Data Availability}

The data used to support the findings of this study are available from the first author and the corresponding author upon request.

\section{Conflicts of Interest}

The authors declare that there are no conflicts of interests regarding the publication of this article.

\section{Acknowledgments}

This work was partially sponsored by the State Key Laboratory of Automotive Safety and Energy under Project No. KF2013, the Joint Laboratory for Internet of Vehicles, Ministry of Education, China Mobile Communications Corporation, under Project No. ICV-KF2018-02, the National Natural Science Foundation of China (No. 51775565), the Tianjin Science and Technology Project (Grant Nos. 16PTGCCX00150, 17ZXRGGX00070, 18YFZCGX00380, and 119YFSLQY00010), the Tianjin Development and Reform Commission Project (Grant No. TJZNZZ1811), the University Foundation of Tianjin University of Technology and Education (Grant No. KYQD 1710), and the Fundamental Research Funds for the Central Universities (Grant No. 18lgpy83). The first author would like to thank Dr. Su L.L., Dr. Guan Z.W., Dr. Hou H.J., Li J.K., Liu X.L. and Tong Y.K. for valuable discussions to improve the quality and presentation of this paper.

\section{References}

[1] J. Andrey, B. Mills, and J. Vandermolen, "A temporal analysis of weather-related collision risk for Ottawa, Canada: 1990-1998," Transportation Research Board, Washington, DC, USA, Paper Number TRB2003-3488, 2003.

[2] R. Attia, J. Daniel, J.-P. Lauffenburger, R. Orjuela, and M. Basset, "Reference generation and control strategy for automated vehicle guidance," in Proceedings of the IEEE Intelligent Vehicles Symposium (IV), pp. 389-394, Madrid, Spain, June 2012.

[3] W. Ben Messaoud, M. Basset, J.-P. Lauffenburger, and R. Orjuela, "Smooth obstacle avoidance path planning for autonomous vehicles," in Proceedings of the IEEE International Conference on Vehicular Electronics and Safety (ICVES), Madrid, Spain, September 2018.
[4] L. Huang, H. C. Guo, R. H. Zhang, and J. P. Wu, "LSTM-based lane-changing behavior model for unmanned vehicle under environment of heterogeneous human-driven and autonomous vehicles," China Journal of Highway and Transport, vol. 33, no. 7, pp. 156-166, 2020.

[5] S. Koenig, M. Likhachev, and D. Furcy, "Lifelong planning A *," Artificial Intelligence, vol. 155, no. 1-2, pp. 93-146, 2004.

[6] A. Gasparetto, P. Boscariol, A. Lanzutti, and R. Vidoni, "Path planning and trajectory planning algorithms: a general overview," Motion and Operation Planning of Robotic Systems, Chapter 1, pp. 3-27, Springer, Cham, Switzerland, 2015.

[7] M. N. Matthew, U. Chris, J. M. Dolan, and J. W. Lee, "Motion planning for autonomous driving with a conformal spatiotemporal lattice," Proc. IEEE International Conference on Robotics and Automation, pp. 4889-4895, 2011.

[8] F. Zhang, J. Gonzales, S. E. Li, F. Borrelli, and K. Li, "Drift control for cornering maneuver of autonomous vehicles," Mechatronics, vol. 54, pp. 167-174, 2018.

[9] A. Stentz, "The focussed D* algorithm for real-time replanning," in Proceedings of the International Joint Conference on Artificial Intelligence, pp. 1652-1659, Montreal, Canada, August 1995.

[10] E. Frazzoli, M. A. Dahleh, and E. Feron, "Real-time motion planning for agile autonomous vehicles," Journal of Guidance, Control, and Dynamics, vol. 25, no. 1, pp. 116-129, 2002.

[11] Y. Liu, "Research on the algorithm optimization of improved ant colony algorithm- LSACA," International Journal of Signal Processing, Image Processing and Pattern Recognition, vol. 9, no. 3, pp. 143-154, 2016.

[12] I. Engedy and G. Horvath, "Artificial neural network based local motion planning of a wheeled mobile robot," in Proceedings of the 11th IEEE International Symposium on Computational Intelligence and Informatics, pp. 213-218, Budapest, Hungary, November 2010.

[13] A. S. Nathan, B. Matthew, R. K. Nitin, C. K. John, and J. C. Gerdes, "Neural network vehicle models for high-performance automated driving," Science Robotics, vol. 4, no. 28, pp. 1-14, 2019.

[14] J.-H. Cho, D.-S. Pae, M.-T. Lim, and T.-K. Kang, "A real-time obstacle avoidance method for autonomous vehicles using an obstacle-dependent Gaussian potential field," Journal of Advanced Transportation, vol. 2018, pp. 1-15, 2018.

[15] H. Xiong, X. Zhu, and R. Zhang, "Energy recovery strategy numerical simulation for dual axle drive pure electric vehicle based on motor loss model and big data calculation," Complexity, vol. 2018, pp. 1-14, 2018.

[16] Q. Tu, H. Chen, and J. Li, "A potential field based lateral planning method for autonomous vehicles," SAE International Journal of Passenger Cars-Electronic and Electrical Systems, vol. 10, no. 1, pp. 24-34, 2017.

[17] B. F. Gomez, F. Cuesta, A. Ollero, and A. Viguria, "Continuous curvature path generation based on $\beta$-spline curves for parking manoeuvers," Robotics and Autonomous Systems, vol. 56, pp. 360-372, 2008.

[18] F. You, R. Zhang, G. Lie, H. Wang, H. Wen, and J. Xu, "Trajectory planning and tracking control for autonomous lane change maneuver based on the cooperative vehicle infrastructure system," Expert Systems with Applications, vol. 42, no. 14, pp. 5932-5946, 2015.

[19] R. H. Zhang, F. You, X. N. Chu, L. Guo, Z. C. He, and R. B. Wang, "Lane change merging control method for unmanned vehicle under V2V cooperative environment," China Journal of Highway and Transport, vol. 31, no. 4, pp. 180-191, 2018. 
[20] K. Kawabata, L. Ma, J. Xue, C. Zhu, and N. Zheng, "A path generation for automated vehicle based on Bezier curve and via-points," Robotics and Autonomous Systems, vol. 74, pp. 243-252, 2015.

[21] M. Wang, S. P. Hoogendoorn, W. Daamen, B. van Arem, and R. Happee, "Game theoretic approach for predictive lanechanging and car-following control," Transportation Research Part C: Emerging Technologies, vol. 58, pp. 73-92, 2015.

[22] X. Sun, H. Zhang, W. Meng, R. Zhang, K. Li, and T. Peng, "Primary resonance analysis and vibration suppression for the harmonically excited nonlinear suspension system using a pair of symmetric viscoelastic buffers," Nonlinear Dynamics, vol. 94, no. 2, pp. 1243-1265, 2018.

[23] T. Peng, L. L. Su, R. H. Zhang, and Z. W. Guan, "A new safe lane-change trajectory model and collision avoidance control method for automatic driving vehicles," Expert Systems with Applications, vol. 141, pp. 1-17, 2019.

[24] C. C. Macadam, "An optimal preview control for linear systems," Journal of Dynamic Systems, Measurement, and Control, vol. 102, no. 3, pp. 188-190, 1980.

[25] R. S. Sharp, D. Casanova, and P. Symonds, "A mathematical model for driver steering control, with design, tuning and performance results," Vehicle System Dynamics, vol. 33, pp. 289-326, 2000.

[26] R.-H. Zhang, Z.-C. He, H.-W. Wang, F. You, and K.-N. Li, "Study on self-tuning tyre friction control for developing main-servo loop integrated chassis control system," IEEE Access, vol. 5, pp. 6649-6660, 2017.

[27] B. Akash, T. Alireza, and B. Shankar, "End-to-end drive bywire PID lateral control of an autonomous vehicle," Proceedings of the Future Technologies Conference, vol. 1, pp. 365-376, 2020.

[28] R. Marino, S. Scalzi, and M. Netto, "Nested PID steering control for lane keeping in autonomous vehicles," Control Engineering Practice, vol. 19, no. 12, pp. 1459-1467, 2011.

[29] S. A. Sangeetha and D. Jean, "An adaptive integral backstepping control approach for UAV," International Journal of Engineering Research \& Technology, vol. 8, no. 5, pp. 485-488, 2019.

[30] P. Wang, S. Gao, L. Li et al., "Automatic steering control strategy for unmanned vehicles based on Robust Backstepping sliding mode control theory," IEEE Access, vol. 7, pp. 1-19, 2019.

[31] A. T. Nguyen, C. Sentouh, and J. C. Popieul, "Fuzzy steering control for autonomous vehicles under actuator saturation: design and experiments," Journal of the Franklin Institute, vol. 355, no. 18, pp. 9374-9395, 2017.

[32] K. L. Che, D. Darshit, and S. Stephen, "Robust model predictive control for autonomous vehicles/self driving cars," Computer Science- Systems and Control, pp. 1-12, 2018.

[33] B. Luca, F. Gianni, M. Matteo, and B. Marco, "LFT-based MPC control of an autonomous vehicle," IFAC-PapersOnLine, vol. 49, no. 15, pp. 7-12, 2016.

[34] M. Zanon, J. V. Frasch, M. Vukov et al., "Model predictive control of autonomous vehicles," Optimization and Optimal Control in Automotive Systems, pp. 41-57, 2014.

[35] R. Billot, N.-E. El Faouzi, and F. De Vuyst, "Multilevel assessment of the impact of rain on drivers' behavior," Transportation Research Record: Journal of the Transportation Research Board, vol. 2107, no. 1, pp. 134-142, 2009.

[36] T. J. Ji, "Effect of rainfall on adhesion coefficient of tirepavement interface," Doctoral thesis, Southeast University, Nanjing, China, 2004.
[37] J. Godthelp, A. R. A. Vanderhorst, and S. Burrij, "Open and closed loop steering in a lane change manoeuvre," Transport Research Board, vol. 1983, pp. 1-36, 1983.

[38] E. F. Camacho and C. B. Alba, Model Predictive Control, Springer Science \& Business Media, Berlin, Germany, 2013. 